\title{
Real Quadratic Fields with Large Class Number
}

\section{Hugh L. Montgomery and Peter J. Weinberger}

Department of Mathematics, Unversity of Michigan, Ann Arbor, Michigan 48104, USA

Let $h, d, \varepsilon$, and $R$ be the class number, discriminant, fundamental unit, and regulator, respectively, of the real quadratic field $\mathbb{Q}(\sqrt{d})$. Let $\chi$ be the primitive quadratic character $(\bmod d)$, and let

$$
L(s, \chi)=\sum_{n=1}^{\infty} \chi(n) n^{-s} .
$$

Then $R=\log \varepsilon$, and

$$
L(1, \chi)=h R d^{-\frac{1}{2}} \text {. }
$$

Since $L(1, \chi) \ll \log d, R>\left(\frac{1}{2}+o(1)\right) \log d$, it follows that $h \ll \sqrt{d}$. Moreover, Littlewood [4] showed that if all nontrivial zeros of $L(s, \chi)$ lie on the critical line $\operatorname{Re} s=\frac{1}{2}$, then $L(1, \chi)<\left(2 e^{\gamma}+o(1)\right) \log \log d$. Hence

$$
h<\left(4 e^{\gamma}+o(1)\right) d^{\frac{1}{2}}(\log d)^{-1} \log \log d
$$

assuming the Generalized Riemann Hypothesis. In this paper we show that the hypothetical estimate (2) can not be improved upon, apart from the value of the constant.

Theorem. There is an absolute constant $c>0$ such that

$$
h>c d^{\frac{1}{2}}(\log d)^{-1} \log \log d
$$

for infinitely many real quadratic fields $\mathbb{Q}(\sqrt{d})$.

To prove the Theorem we construct $d$ for which $R<\log d$, and $L(1, \chi)>$ $c \log \log d$. Then (3) follows from (1). We consider only square-free $d$ with $d \equiv 1(\bmod 4), d=n^{2}+1$. Then $\varepsilon=n+\sqrt{d}<2 \sqrt{d}<d$, and so $R<\log d$. To make $L(1, \chi)$ large we wish to have $\chi(p)=1$ for many small primes $p$. By quadratic reciprocity this amounts to having $d$ lie in certain arithmetic progressions. Following an argument of Estermann [3], we show in Lemma 1 that such $d$ exist. Then in Lemma 2 we relate $L(1, \chi)$ to $\chi(p)$ for small $p$. With these lemmas established, it is then a simple matter to complete the proof of the Theorem. 
Lemma 1. Let $D(x ; q, a)$ denote the number of $d \leqq x$ such that $d$ is square-free, $d=n^{2}+1$ for some integer $n$, and $n \equiv a(\bmod q)$. Suppose that $2 \mid q$, and that $\left(a^{2}+1, q\right)=1$. Then

$$
D(x ; q, a)=\frac{x^{\frac{1}{2}}}{q} \prod_{p \nmid q}\left(1-2 p^{-2}\right)+O\left(x^{-\frac{2}{3}} \log x\right) .
$$

Proof. Clearly

$$
\begin{aligned}
D(x ; q, a) & =\sum_{\substack{n \leqq(x-1)^{1 / 2} \\
n \equiv a(\bmod q)}} \sum_{r^{2} \mid\left(n^{2}+1\right)} \mu(r) \\
& =\sum_{r \leqq x} \mu(r) \sum_{\substack{n \leqq(x-1)^{1 / 2} \\
n \equiv a(\bmod q) \\
\mathbf{r}^{2} \mid\left(n^{2}+1\right)}} 1 .
\end{aligned}
$$

If $(q, r)>1$ then the inner sum vanishes, since $\left(n^{2}+1, q\right)=1$ for $n \equiv a(\bmod q)$. Thus we may suppose that $(q, r)=1$. We consider $r \leqq y, y<r \leqq x$ separately. Writing $n^{2}+1=r^{2} s$, we see that

$$
\sum_{y<r \leqq x} \mu(r) \sum_{\substack{n \leqq(x-1)^{1 / 2} \\ n=a(\bmod q) \\ r^{2} \mid\left(n^{2}+1\right)}} 1 \ll \sum_{s \leqq x y^{-2}} \sum_{\substack{n, r \\ r^{2} s=n^{2}+1}} 1 .
$$

From the theory of Pell's equation, the number of pairs $u, v$ for which $u^{2}-s v^{2}=-1$, $1 \leqq u \leqq U$, is $\ll \log U$, uniformly in $s$. Thus the inner sum above is $\ll \log x$, and so the contribution of $r>y$ is $\ll x y^{-2} \log x$. Thus

$$
D(x ; q, a)=\sum_{\substack{r \leqq y \\(q, r)=1}} \mu(r) \sum_{\substack{n \leqq(x-1)^{1 / 2} \\ n=a(\bmod q) \\ r^{2} \mid\left(n^{2}+1\right)}} 1+O\left(x y^{-2} \log x\right) .
$$

For odd $m$ the number of solutions $n(\bmod m)$ of the congruence

$$
n^{2}+1 \equiv 0(\bmod m) \text { is } \prod_{p \mid m}\left(1+\left(\frac{-1}{p}\right)\right)=c(m), \text { say. }
$$

But $2 \mid q$ and $(q, r)=1$, so $r$ is odd, and so the number of $n\left(\bmod q r^{2}\right)$ for which $r^{2} \mid\left(n^{2}+1\right), n \equiv a(\bmod q)$, is $c\left(r^{2}\right)=c(r)$. Hence the inner sum above is $=c(r)\left(x^{\frac{1}{2}} q^{-1} r^{-1}+O(1)\right)$. Now $c(r) \leqq d(r)$ so

$$
\sum_{r \leqq y} c(r) \ll y \log y, \quad \text { and } \sum_{r>y} c(r) r^{-2} \ll y^{-1} \log y \text {. }
$$

Therefore

$$
\begin{aligned}
D(x ; q, a)= & \frac{x^{\frac{1}{2}}}{q} \sum_{\substack{r=1 \\
(q, r)=1}}^{\infty} \mu(r) c(r) r^{-2}+O(y \log y)+O\left(x^{\frac{1}{2}} q^{-1} y^{-1} \log y\right) \\
& +O\left(x y^{-2} \log x\right) .
\end{aligned}
$$

Taking $y=x^{\frac{2}{3}}$, we obtain the result, since the sum over $r$ is

$$
=\prod_{p \Upsilon_{q}}\left(1-\left(1+\left(\frac{-1}{p}\right)\right) p^{-2}\right) \text {. }
$$


Lemma 2. Suppose that $0<\delta<1$. Then for $(\log q)^{\delta} \leqq y \leqq \log q$, and $\chi$ a primitive character $(\bmod q), q>1$,

$$
\log L(1, \chi)=\sum_{p \leqq y} \chi(p) p^{-1}+O_{\delta}(1)
$$

unless $\chi$ lies in an exceptional set $\mathfrak{E}(\delta)$. The set $\mathfrak{E}(\delta)$ contains $\ll Q^{\delta}$ primitive characters $\chi$ with conductor $q \leqq Q$.

A more precise result of this sort has been given by Elliott [2]; for the sake of completeness we include a short proof of Lemma 2.

Proof. Clearly

$$
\sum_{n \leqq x} \chi(n) \Lambda(n)(n \log n)^{-1}=\frac{1}{2 \pi i} \int \log L(s, \chi) \frac{x^{s-1}}{s-1} d s,
$$

where the contour is the straight line from $c-i \infty$ to $c+i \infty, c>1$. Let $\mathbb{E}$ be the set of primitive characters $\chi$ for which $L(s, \chi)$ has at least one zero in the rectangle

$$
1-\frac{1}{7} \delta \leqq \sigma \leqq 1, \quad|t| \leqq(\log q)^{2} .
$$

Suppose that $\chi \notin \mathfrak{E}$. Arguing in the usual manner (see Titchmarsh [8, Lemma 3.12]), we see that the portion of the above integral for which $|t| \geqq \log q$ contributes $\ll 1$, uniformly for $x \leqq q$. For $|t| \leqq \log q$ we take the contour to the abcissa $\sigma=1-\frac{1}{8} \delta$, passing the pole at $s=1$ (with residue $\log L(1, \chi)$ ). We may neglect higher powers of primes with error $\ll 1$, so

$$
\sum_{p \leqq x} \chi(p) p^{-1}-\log L(1, \chi) \ll 1+\int_{-\log q}^{\log q}\left|\log L\left(1-\frac{1}{8} \delta+i t, \chi\right)\right| \frac{d t}{\delta+|t|} .
$$

But $L(s, \chi) \neq 0$ for $s$ in the rectangle (4), which implies that $\log L(s, \chi) \ll_{\delta} \log q$ in the integrand above. Hence the above is $\ll 1+x^{-\frac{1}{8} \delta}(\log q)^{2}$. Taking $x=(\log q)^{16 \delta^{-1}}$, we find that

$$
\log L(1, \chi)-\sum_{p \leqq y} \chi(p) p^{-1} \ll_{\delta} 1+\sum_{y<p \leqq x} p^{-1} \ll_{\delta} 1,
$$

since $y \geqq(\log q)^{\delta}$.

It remains now to estimate the number of characters lying in the set $\mathfrak{E}=\mathfrak{E}(\delta)$. Let $N(\sigma, T, \chi)$ denote the number of zeros $\varrho=\beta+i \gamma$ of $L(s, \chi)$ in the rectangle $\sigma \leqq \beta \leqq 1,|\gamma| \leqq T$. From a theorem of Montgomery [6] (see also [7, Theorem 12.2] or [1, Théorème 20]),

$$
\sum_{q \leqq Q} \sum_{\chi}^{*} N(\sigma, T, \chi) \ll\left(Q^{2} T\right)^{3(1-\sigma)}(\log Q T)^{9} .
$$

Here $\sum_{x}^{*}$ denotes a sum over all primitive characters $\chi(\bmod q)$. Thus the number of zeros in question is $\ll Q^{\delta}$, so Lemma 2 is established.

We now complete the proof of the Theorem. Let $y=\frac{1}{9} \log x, q=2 \prod_{p \leqq y} p, a=0$.

Then $q<x^{\frac{1}{\phi}}, 2 \mid q,\left(a^{2}+1, q\right)=1$, and if $d=n^{2}+1, n \equiv a(\bmod q)$, then $\chi(p)=\left(\frac{d}{p}\right)=1$ 
for all $p \leqq y$. By Lemma 1 there are $\gg x^{\frac{1}{2}} q^{-1} \gg x^{\frac{3}{8}}$ such square-free $d \leqq x$. From Lemma 2 , with $\delta<\frac{3}{8}$, we see that $L(1, \chi)>c_{1} \log y>c \log \log d$ for almost all of these $d$. This completes the proof of the Theorem.

\section{References}

1. Bombieri, E.: Le grand crible dans la théorie analytique des nombres. Astérisque 18 , Société Math. de France 1974

2. Elliott, P.D.T.A.: The distribution of the quadratic class number. Litovsk. Mat. Sb. 10, 189-196 (1970)

3. Estermann, T.: Einige Sätze über quadratfreie Zahlen. Math. Ann. 105, $653-662$ (1931)

4. Littlewood,J.E.: On the class number of the corpus $P(\sqrt{-k})$. Proc. London Math. Soc. 27, $358-372(1927)$

5. Montgomery, H.L.: Zeros of $L$-functions. Invent. Math. 8, 346-354 (1969)

6. Montgomery, H.L.: Topics in multiplicative number theory. Lecture Notes in Mathematics 227. Berlin, Heidelberg, New York: Springer 1971

7. Titchmarsh, E.C.: The theory of the Riemann zeta-function. Oxford: Oxford University Press 1951

Received July 6, 1976 